\title{
Ellipsometric Evaluation of the Optical Constants of Zirconium Oxynitride Thin Films Deposited by Reactive Pulsed Magnetron Sputtering
}

\begin{abstract}
I.B.I. TOMSAH
Physics Department, College of Science, Qassim University, P.O. 6644, 51452, Buryadh, Kingdom of Saudi Arabia

(Received June 12, 2012; in final form March 5, 2013)

Three different zirconium oxynitride films were deposited onto glass and $\mathrm{Si}(100)$ substrates at room temperature by pulsed reactive dc magnetron sputtering of a metallic $\mathrm{Zr}$ target in an $\mathrm{Ar} / \mathrm{O}_{2} / \mathrm{N}_{2}$ atmosphere. The structural, compositional and optical properties of the deposited films were found to depend on the ratio of nitrogen partial pressure to the total reactive gas partial pressure. Energy-dispersive X-ray spectroscopy measurements revealed that as the nitrogen amount increased in the reactive gas the nitrogen content was found to increase in the film. The film structure was determined by X-ray diffraction. The X-ray diffraction patterns of the analyzed samples revealed a strong dependence of the $\mathrm{ZrO}_{x} \mathrm{~N}_{y}$ film structure on composition. A two layer model, the Bruggeman effective medium approximation and both Drude absorption edge and Lorentz oscillators were used to describe the surface roughness layer and the main $\mathrm{ZnO}_{x} \mathrm{~N}_{y}$ layer, respectively, was used to describe the experimental ellipsometric data. The optical band gap was decreased from 3.56 to $3.45 \mathrm{eV}$ with changing nitrogen content, while refractive index at $650 \mathrm{~nm}$ simultaneously was increased from 1.98 to 2.11 .
\end{abstract}

DOI: $10.12693 /$ APhysPolA.124.141

PACS: 81.15.Gh, 61.05.C-, 78.66.-w

\section{Introduction}

Transition metal oxynitride thin films have recently attracted the attention of many researchers, because of its versatile properties [1-4]. This is because the presence of oxygen in nitride compounds leads to unexpected and promising functional range materials.

Cheng and Thompson [5] found another $\mathrm{Zr}-\mathrm{O}-\mathrm{N}$ phase called $\beta^{\prime \prime}$. Lerch et al. $[6,7]$ showed that oxynitrides with a high ionic conductivity, comparable with yttria stabilized cubic $\mathrm{ZrO}_{2}$, could be obtained by a high temperature reaction of $\mathrm{ZrO}_{2}$ with nitrogen. These oxynitrides of zirconium were prepared by nitridation of commercial $\mathrm{ZrO}_{2}$ powder in a graphite-heated resistance furnace in a nitrogen atmosphere of 1 bar and temperatures higher than $1700^{\circ} \mathrm{C}$ [8] and had numerous applications.

Zirconium dioxide and $\mathrm{ZrO}_{2} \mathrm{Y}_{2} \mathrm{O}_{3}$ due to their optical properties show many applications in optical components like when high laser power is used in order to minimize the height pulse laser damage threshold and in this cases doping the coatings with nitrogen can improve the refractive index and the protection $[9,10]$. These kind of coatings have a lot of other applications, such as buffer layers for growing superconductors, oxygen sensors, in situations where thermal isolation and chemical protection (TBCs) are required, in bio-applications, etc. [11-15].

$\mathrm{ZrO}_{2}$ base coatings have been deposited by many techniques but in particular physical vapour deposition (PVD - magnetron sputtering) presents a powerful way for depositing thin films with high quality and good relation of properties because of its ability to control the addition, at an atomistic level, of stabilizing materials, and by changing the deposition parameters it is possible to tailor the properties, which are the most important for each application [11, 16-21].
The incorporation of nitrogen on the structure of ceramics can improve the fracture toughness [22], and zirconium oxynitride presents also the high oxide ion conductivity [23, 24].

The aim of the present work is to study the influence of the deposition condition, such as the reactive gas mixture flow on some of the properties of magnetron sputtered $\mathrm{ZrO}_{x} \mathrm{~N}_{y}$ thin films. In addition, the influence of $\mathrm{N}_{2}$ incorporation on the lattice of the $\mathrm{ZrO}_{2}$ coatings is performed in order to study the effect on the structure and optical properties. Changes induced by the variation of the reactive gas mixture composition on the compositional and structural properties have been studied.

\section{Experimental procedure}

$\mathrm{ZrO}_{x} \mathrm{~N}_{y}$ films were prepared on both glass slides and $\mathrm{Si}$ wafers $\langle 100\rangle$ as substrates at room temperature by pulsed dc reactive magnetron sputtering of a metallic zirconium target in an argon, oxygen and nitrogen gas mixtures. A metal target with $7.5 \mathrm{~cm}$ diameter was used with an average power of $550 \mathrm{~W}$. The target-to-substrate distance was $10 \mathrm{~cm}$. Before deposition the substrates were chemically etched using diluted HF to remove the native oxide layer, ultrasonically cleaned in acetone and then dried by blowing nitrogen. Before deposition, the chamber was evacuated to $1.3 \times 10^{-4} \mathrm{~Pa}$ at full pumping speed of $1500 \mathrm{l} / \mathrm{s}$ for air. To accommodate the relatively high pressure during sputtering, the pumping speed was throttled to about $25 \%$ of its maximum, resulting in a throttled base pressure of about $1.3 \times 10^{-3} \mathrm{~Pa}$.

The total gas pressure during deposition process was kept constant at $0.4 \mathrm{~Pa}$ as monitored by a Baratron ${ }^{\circledR}$ capacitance manometer, by adjusting the Ar flow while systematically varying the $\mathrm{O}_{2}$ and $\mathrm{N}_{2}$ partial pressures. 
A differentially pumped gas monitor (PPM 100 by SRS) was used to measure the partial pressures during deposition. This gas monitor was pre-calibrated via the readings of the Baratron.

Oxygen partial pressure $\left(P_{\mathrm{O}_{2}}\right)$ of $5 \times 10^{-2} \mathrm{~Pa}$ was used to prepare $\mathrm{ZrO}_{x} \mathrm{~N}_{y}$ films. At this oxygen partial pressure, the $\mathrm{Zr}$ target operated in the "poisoned" mode (oxide layer was present). Next, the $\mathrm{O}_{2}$ flow was partially replaced by $\mathrm{N}_{2}$ flow. The partial pressure of $\mathrm{N}_{2}$ in the reactive gas mixture to the total reactive gas, $\Gamma=P_{\mathrm{N}_{2}} /\left(P_{\mathrm{N}_{2}}+P_{\mathrm{O}_{2}}\right)$, was adjusted to be $0.03,0.85$, and 0.98 .

Two different kinds of substrates were used to get the best accuracy from each measurement. The glass slides are characterized by an amorphous structure. The glass slides were used for X-ray diffraction (XRD) because of their amorphous structure. The Si (100) substrates are characterized by very smooth surfaces and they do not contain oxygen in their chemical composition. Therefore, they were used in energy-dispersive X-ray spectroscopy (EDAX) and ellipsometric measurements.

EDAX bulk composition measurements were performed in a Philips XL 30 scanning electron microscope at $10 \mathrm{kV}$ using only internal absorption values and, therefore, the oxygen and nitrogen content could be higher than that of the figures obtained. The accuracy of EDAX in determining oxygen or nitrogen could be less than at 4 at.\%.

The structural study was carried out by XRD with a Philips difractometer $\mathrm{PW}-170$ with a $\mathrm{Cu} K_{\alpha}$ radiation $(\lambda=1.54056 \AA)$ and changing the $2 \theta$ angle between $20^{\circ}$ and $80^{\circ}$ with steps of $0.02^{\circ}$.

Variable angle spectroscopic ellipsometry (VASE) data for $\mathrm{ZnO}_{x} \mathrm{~N}_{y}$ films prepared on $\mathrm{Si}$ (100) were acquired using a PHE-102 variable angle spectroscopic ellipsometer (Angstrom Advanced Inc.) in the wavelength range of $300-1500 \mathrm{~nm}$. The data were acquired at angle of incidences of $55^{\circ}, 60^{\circ}$ and $65^{\circ}$. The instrument measures the complex ratio of the Fresnel reflection coefficients for $p$ - and $s$-polarized light and reports the ratio in terms of the ellipsometric parameters $\psi$ and $\Delta$ defined by the equation

$$
F=\tan (\psi) \exp (\mathrm{i} \Delta)=\tilde{r}_{p} / \tilde{r}_{s},
$$

where $\tilde{r}_{p}$ and $\tilde{r}_{s}$ are the amplitude reflection coefficient for light polarized in the $p$ - and $s$-plane of incidence, respectively. The data obtained from the ellipsometer were accurately modeled using the PHE-102 software package. Ellipsometric data $\psi$ and $\Delta$ for variable wavelengths were fitted in the optical model.

\section{Results and discussion}

\subsection{EDAX and XRD examinations}

Figure 1a-c depicts typical EDAX spectra recorded for the three $\mathrm{ZrO}_{x} \mathrm{~N}_{y}$ samples for different atomic concentrations of nitrogen and oxygen, as tabulated in Table. The EDAX analysis confirms the presence of nitrogen, oxygen and zirconium elements in the deposited films. In addition, signals from silicon and carbon elements, which presumably could appear from the silicon substrate and the

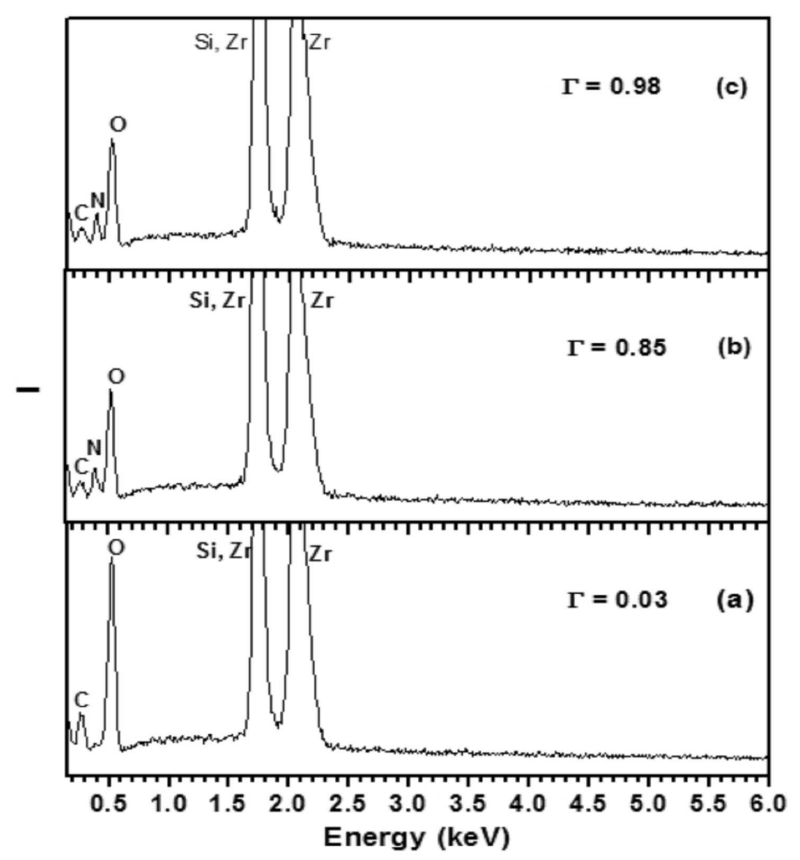

Fig. 1. EDAX spectra of $\mathrm{ZrO}_{x} \mathrm{~N}_{y}$ films deposited at different $\Gamma$ values.

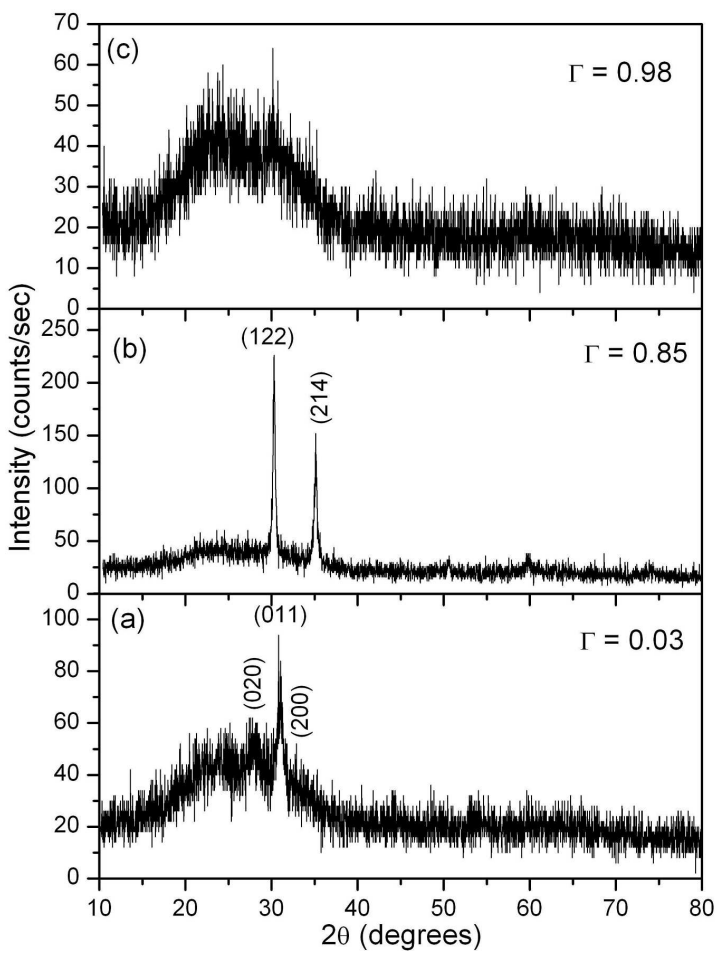

Fig. 2. XRD patterns of $\mathrm{ZrO}_{x} \mathrm{~N}_{y}$ films produced on the glass substrates for the different $\Gamma$ values.

carbon contamination, respectively, as observed in the same figure. Upon increasing the $\Gamma$ values, the amount of nitrogen in the film is increased and this can be clearly seen from the increase in the intensities of the nitrogen characteristic peak. 
XRD measurements were performed in order to determine the crystal structure of the films and to identify a possible transformation in the analyzed samples. Figure $2 \mathrm{a}-\mathrm{c}$ shows the XRD patterns of the sputtered $\mathrm{ZrO}_{x} \mathrm{~N}_{y}$ films with $\Gamma$ values of $0.03,0.85$ and 0.98 . For the lower $\Gamma$ value of 0.03 (lower nitrogen content), the pattern exhibits two diffraction peaks at $2 \theta$ of three peaks at $2 \theta$ of $27.4^{\circ}, 30.4^{\circ}$ and $32.8^{\circ}$. These peaks can be in- dexed as zirconium oxide orthorhombic-type structure (JCPDS card no. 83-0810). The increase of the nitrogen reactive gas flow rates is followed by the appearance of two clearly distinguished diffraction peaks (around $2 \theta$ of $30.3^{\circ}$ and $35.2^{\circ}$ ), which may be assigned to the (122) and (214) of a zirconium oxide nitride (JCPDS card no. 48-1637). Up on increasing the $\Gamma$ to 0.98 , an amorphous structure is observed.

Thickness, RMS roughness and chemical composition of $\mathrm{ZrO}_{x} \mathrm{~N}_{y}$ films as a function of $\Gamma$ values.

TABLE

\begin{tabular}{c|c|c|c|c|c|c|c|c}
\hline \hline$\Gamma$ & $\begin{array}{c}\text { Thickness determined } \\
\text { from simulations } \\
{[\mathrm{nm}]}\end{array}$ & $\begin{array}{c}\text { RMS } \\
\text { roughness } \\
{[\mathrm{nm}]}\end{array}$ & $\begin{array}{c}\text { Refractive } \\
\text { at } \\
650 \mathrm{~nm}\end{array}$ & $\begin{array}{c}\text { Band gap, } \\
E_{\mathrm{g}}[\mathrm{eV}]\end{array}$ & \multicolumn{2}{|c|}{$\begin{array}{c}\text { Chemical composition } \\
\text { atomic [\%] }\end{array}$} & \multirow{2}{*}{$\mathrm{ZrO}_{x} \mathrm{~N}_{y}$} \\
\cline { 7 - 8 } & 285.7 & 3.2 & 1.98 & 3.56 & 31.44 & 68.56 & 0.0 & $\mathrm{ZrO}_{2.18}$ \\
0.03 & 307.8 & 3.9 & 2.05 & 3.49 & 31.18 & 60.11 & 8.71 & $\mathrm{ZrO}_{1.93} \mathrm{~N}_{0.28}$ \\
0.98 & 303.1 & 4.1 & 2.11 & 3.45 & 30.98 & 51.18 & 17.84 & $\mathrm{ZrO}_{1.65} \mathrm{~N}_{0.58}$
\end{tabular}

\subsection{Ellipsometric results and modulation}

The choice of the appropriate optical model plays a major role in the evaluation of the optical constants from the ellipsometric spectra (SE). Thus, the refractive index $(n)$ and extinction coefficient $(k)$ of $\mathrm{ZrO}_{x} \mathrm{~N}_{y}$ films are obtained by assuming a two-layer model, representing the film and the surface roughness layer. The complex refractive index of the film is described by a Lorentz oscillator's model. The complex refractive index of the surface roughness layer is calculated by the Bruggeman effective medium approximation (BEMA) assuming a mixture of the "Cauchy-material" and a fitted percentage (volume fraction) of voids (air). From a Lorentz oscillator model, the complex dielectric function can be expressed by the following relation [25]:

$$
\varepsilon(h \nu)=\varepsilon_{\infty}+\sum_{j=1}^{N} \frac{A_{j}}{E_{j}^{2}-(h \nu)^{2}-\mathrm{i} B_{j} h \nu},
$$

where $A_{j}$ is the amplitude, $E_{j}$ - the center energy of $j$-th oscillator, $B_{j}$ - the broadening of each oscillator, $h \nu$ the photon energy in $\mathrm{eV}$ and $\varepsilon_{\infty}$ - the dielectric function at infinite (contribution from the higher-frequency oscillators). The four terms $\left(\varepsilon_{\infty}, A_{j}, E_{j}\right.$, and $\left.B_{j}\right)$ are used as fit parameters. The centre energy of the first oscillator is fixed at zero, for the film with $\Gamma=0.98$, to describe free carriers based on the Drude model. The thickness and the voids fraction of the BEMA surface layer and the thickness of the interface layer are also selected as fit parameters. An example for the best fit of the proposed models compared with the measured ellipsometric data of the films prepared at $\Gamma=0.85$ is shown in Fig. $3 \mathrm{a}-\mathrm{c}$. It is seen that there is a good agreement between the simulated and the experimental data. Thus, the optical constants can be adequately extracted. It is observed that the surface roughness increases slightly with increasing $\Gamma$ values.

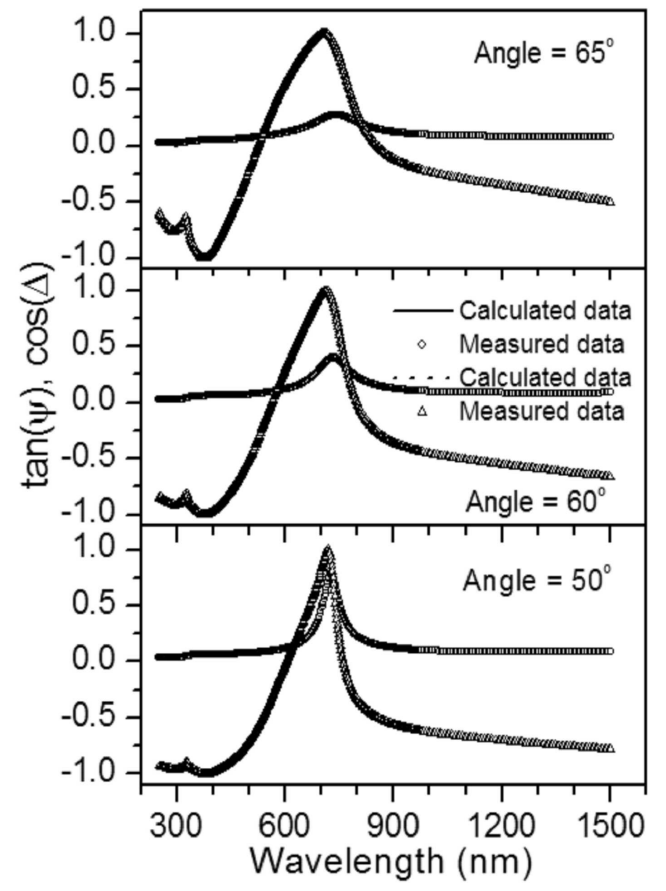

Fig. 3. Experimental and calculated ellipsometric $\psi$ and $\Delta$ spectra at $55^{\circ}, 60^{\circ}$, and $65^{\circ}$ angles of incidence. The spectra are for the film with $\Gamma=0.85$.

The variations of refractive index, $n$, and extinction coefficient, $k$, as a function of wavelength for $\mathrm{ZrO}_{x} \mathrm{~N}_{y}$ films prepared at various $\Gamma$ are plotted in Fig. 4 and Fig. 5, respectively. The refractive index of the films increases upon increasing nitrogen incorporation i.e. $\Gamma$ values. In other words the calculated refractive index values are found to depend on oxygen and nitrogen flow. The calculated values for the refractive index $n$ at $650 \mathrm{~nm}$ for $\mathrm{ZrO}_{x} \mathrm{~N}_{y}$ films are given in Table. The refractive index at $650 \mathrm{~nm}$ of the $\mathrm{ZrO}_{x} \mathrm{~N}_{y}$ films are comparable to those 


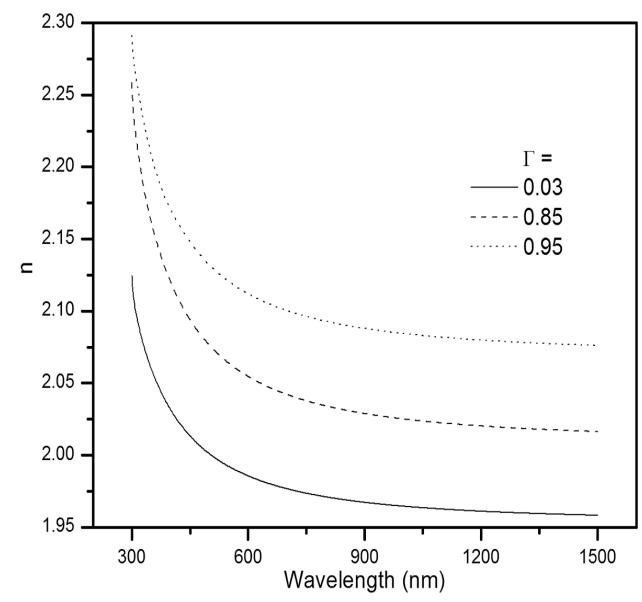

Fig. 4. Refractive index as a function of wavelength of $\mathrm{ZrO}_{x} \mathrm{~N}_{y}$ films with different $\Gamma$ values.

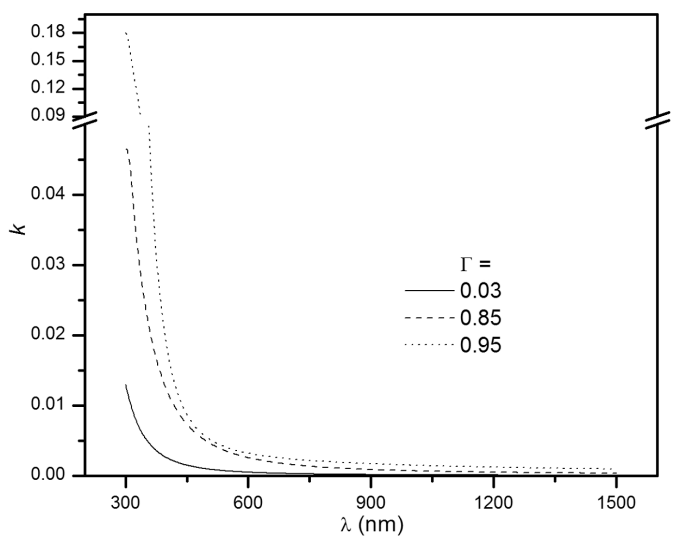

Fig. 5. Extinction coefficient as a function of wavelength of $\mathrm{ZrO}_{x} \mathrm{~N}_{y}$ films with different $\Gamma$ values.

obtained by Venkataraj et al. [26] for $\mathrm{ZrO}_{x} \mathrm{~N}_{y}$ films prepared by dc reactive magnetron sputtering.

The extinction coefficient is found to increase with increasing $\Gamma$ values. Over the wavelength region from $450 \mathrm{~nm}$ to $1500 \mathrm{~nm}$ the extinction coefficient, $k$, of the films is very close to zero. The $k$ values increase strongly with wavelength for the wavelengths greater than $450 \mathrm{~nm}$. The dramatic increases in $n$ and $k$ values, with decreasing wavelength, in the shorter wavelength region $(\lambda<450 \mathrm{~nm})$ are due to the onset of electronic transitions through the energy gap of the $\mathrm{ZrO}_{x} \mathrm{~N}_{y}$ [27].

The absorption coefficient $(\alpha)$ was calculated using the obtained $k$ values from the relation

$$
\alpha=\frac{4 \pi k}{\lambda} \text {. }
$$

The optical band gap $\left(E_{\mathrm{g}}\right)$ values is calculated according to a direct allowed transition from the general relation

$$
(\alpha h \nu) \propto\left(h \nu-E_{\mathrm{g}}\right)^{1 / 2} .
$$

The $E_{\mathrm{g}}$ of $\mathrm{ZrO}_{x} \mathrm{~N}_{y}$ films values are calculated from the intercept of the linear fit with the energy axis, as shown

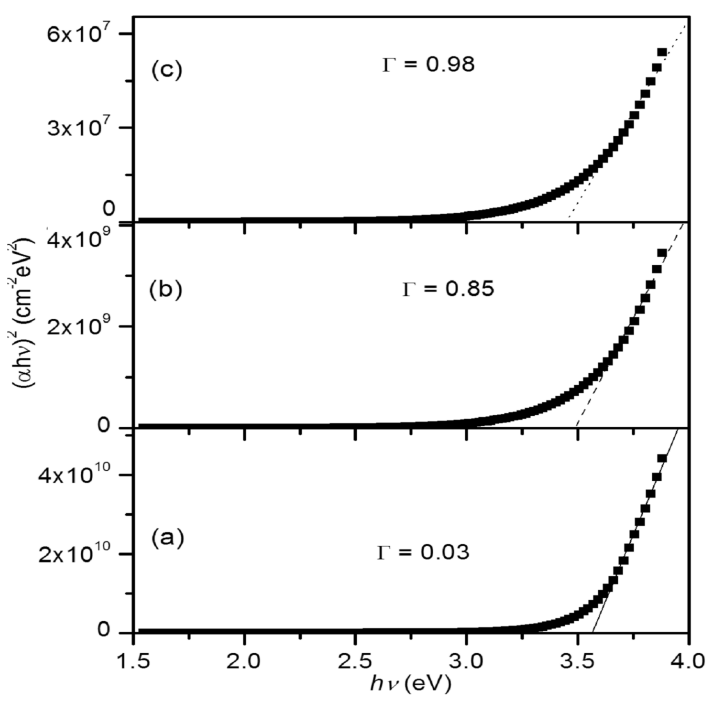

Fig. 6. Plot of $(\alpha h \nu)^{2}$ versus photon energy $h \nu$ for transition $\mathrm{ZrO}_{x} \mathrm{~N}_{y}$ films for the different $\Gamma$.

in Fig. 6. The obtained $E_{\mathrm{g}}$ values are listed in Table. For pure zirconium oxide films a band gap of approximately $3.56 \mathrm{eV}$ is determined, that is nearly $66 \%$ of the calculated value of monoclinic zirconium oxide $(5.42 \mathrm{eV})$ by Karlik et al. using the method of Hybertsen and Louie [28]. Also, it is lower than the value $4.96 \mathrm{eV}$ reported by Zhu et al. [29] for $\mathrm{ZnO}_{2}$ films prepared by nitrogen-assisted reactive magnetron sputtering, however is close to the value $3.67 \mathrm{eV}$ reported by Mohamed et al. for $\mathrm{ZrO}_{2}$ films prepared by reactive pulsed magnetron sputtering [30] and to the value $3.85 \mathrm{eV}$ reported by Korkmaz et al. [31] for $\mathrm{ZrO}_{2}$ thin films deposited by thermionic vacuum arc. This low band gap value, comparing to other published work, may be ascribed to the different preparation conditions and to the deposition stresses [32]. Upon increasing $\Gamma$ value the band gap of the $\mathrm{ZrO}_{x} \mathrm{~N}_{y}$ films decreases slowly down to $3.49 \mathrm{eV}$ and then to $3.45 \mathrm{eV}$ at $\Gamma=0.85$ and $\Gamma=0.98$, respectively. The decrease in $E_{\mathrm{g}}$ is attributed to the formation of well localized $\mathrm{N} 2 p$ states above the $\mathrm{O} 2 p$ valence band states. Interrelation of such energy levels in the band gap reduces the band gap and increases the visible light absorption through a charge transfer between a dopant and a conduction or valence band [33].

\section{Conclusions}

Zirconium oxynitride films have been deposited using reactive pulsed magnetron sputtering in $\mathrm{N}_{2}+\mathrm{O}_{2}+\mathrm{Ar}$ atmosphere. The crystal structure depends on the $\Gamma$ values i.e. nitrogen content. The variation in band gap of zirconium oxynitride films from 3.45 to $3.56 \mathrm{eV}$ is observed, which is between 64 and $66 \%$ of calculated value of bulk $\mathrm{ZrO}_{2}(5.42 \mathrm{eV})$ at different nitrogen partial pressures. The refractive index and the extinction coefficient 
are found to increase, whereas the optical band gap decreases with increasing nitrogen content in the film.

\section{Acknowledgments}

The author would like to thank Dr. Sodky H. Mohamed for his technical support and fruitful discussions.

\section{References}

[1] S. Collard, H. Kupfler, G. Hecht, W. Hoyer, H. Moussaoui, Surf. Coat. Technol. 112, 181 (1999).

[2] Y. Saito, M. Hirata, H. Tada, M. Hyodo, Appl. Phys. Lett. 63, 1319 (1993).

[3] G. Hitoki, T. Takata, J. Kondo, M. Hara, H. Kobayashi, K. Domen, Chem. Commun. $\mathbf{1 6}$, 1698 (2002).

[4] E. Vogelzang, J. Sjollema, H.J. Boer, J.T.M. Doer Hosson, J. Appl. Phys. 61, 4606 (1987).

[5] Y.B. Cheng, D.P. Thompson, Spec. Ceram. 9, 149 (1992).

[6] M. Lerch, F. Krumeich, R. Hock, Solid State Ionics 95, 87 (1997).

[7] J. Wendel, M. Lerch, W. Laqua, J. Solid State Chem. 142, 163 (1999).

[8] M.A. Signore, A. Rizzo, L. Mirenghi, M.A. Tagliente, A. Cappello, Thin Solid Films 515, 6798 (2007).

[9] T. Sikola, J. Spousta, L. Dittrichova, I. Benes, Nucl. Instrum. Methods Phys. Res. B 127-128, 673 (1999).

[10] M.M. Mikhailov, V.V. Neshchimenko, C. Li, B.-J. Ye, J. Lumin. 130, 1671 (2010).

[11] V. Teixeira, M. Andritschky, W. Fischer, H.P. Buchkremer, D. Stöver, Surf. Coat. Technol. 120-121, 103 (1999).

[12] G.Z. Cao, H.W. Brinkman, J. Meijerink, K.J. De Vries, A. Burggraaf, J. Am. Ceram. Soc. 76, 2201 (1993).

[13] J.T. DeMasi-Marcin, K. Gupta Dinesh, Surf. Coat. Technol. 68/69, 1 (1994).

[14] G. Johner, K.K. Schweitzer, J. Vac. Sci. Technol. A 3, 2516 (1985).
[15] A. Portinha, V. Teixeira, Vacuum 82, 1517 (2008).

[16] R.C. Garvie, J. Phys. Chem. 82, 218 (1978).

[17] V. Teixeira, A. Monteiro, J. Duarte, A. Portinha, Vacuum 67, 477 (2002).

[18] R. Brenier, A. Gagnair, Thin Solid Films 392, 142 (2001).

[19] J.P. Holgado, J.P. Espino's, F. Yubero, A. Justo, M. Ocanã, J. Benıtez, A.R. González-Elipe, Thin Solid Films 389, 34 (2001).

[20] L. Bianchi, A.C. Leger, H. Vardelle, A. Vardelle, P. Fauchais, Thin Solid Films 305, 35 (1997).

[21] K. Goedicke, J. Liebig, O. Zywitzki, H. Sahm, Thin Solid Films 377-378, 37 (2000).

[22] J. Wrba, M. Lerch, G. Muller, J. Mater. Sci. Lett. 19, 107 (2000).

[23] M. Ohashi, H. Yamamoto, S. Yamanaka, M. Hattori, Mater. Res. Bull. 28, 513 (1993).

[24] M. Lerch, J. Mater. Sci. Lett. 17, 441 (1998).

[25] R.A. Synowicki, Thin Solid Films 313-314, 394 (1998).

[26] S. Venkataraj, O. Kappertz, R. Jayavel, M. Wuttig, J. Appl. Phys. 92, 2461 (2002).

[27] F. Rubio, J.M. Albella, J. Denis, J.M. MartnezDuart, J. Vac. Sci. Technol. 21, 1043 (1982).

[28] B. Karlik, E.K. Chang, S.G. Louie, Phys. Rev. B 57 , 7027 (1998)

[29] L.Q. Zhu, Q. Fang, G. He, M. Liu, L.D. Zhang, Nanotechnology 16, 2865 (2005).

[30] S.H. Mohamed, A.M. Abd El-Rahman, M.R. Ahmed, J. Phys. D: Appl. Phys. 40, 7057 (2007).

[31] S. Korkmaz, S. Pat, N. Ekem, M.Z. Balbag, S. Temel, Vacuum 86, 1930 (2012).

[32] S.H. Mohamed, A.M. Abd El-Rahman, A.M. Salem, L. Pichon, F.M. El-Hossary, J. Phys. Chem. Solids 67, 2351 (2006).

[33] C. Di Valentin, G. Pacchioni, A. Selloni, Phys. Rev. B 70, 085116 (2004). 